\title{
Strong-Field Double Ionization of Ar below the Recollision Threshold
}

\author{
Yunquan Liu, ${ }^{1}$ S. Tschuch, ${ }^{1}$ A. Rudenko, ${ }^{1}$ M. Dürr, ${ }^{1}$ M. Siegel,${ }^{2}$ U. Morgner, ${ }^{2}$ R. Moshammer, ${ }^{1}$ and J. Ullrich ${ }^{1}$ \\ ${ }^{1}$ Max-Planck-Institut für Kernphysik, Saupfercheckweg 1, D-69117 Heidelberg, Germany \\ ${ }^{2}$ Leibniz Universität Hannover, Welfengarten 1, D-30167 Hannover, Germany \\ (Received 22 January 2008; published 28 July 2008)
}

\begin{abstract}
Nonsequential double ionization of Ar by 45 fs laser pulses $(800 \mathrm{~nm})$ at $(4-7) \times 10^{13} \mathrm{~W} / \mathrm{cm}^{2}$ was explored in fully differential measurements. Well below the field-modified recollision threshold we enter the multiphoton regime. Strongly correlated back-to-back emission of the electrons along the polarization direction is observed to dominate in striking contrast to all previous data. No effect of Coulomb repulsion can be found, the predicted cutoff in the sum-energy spectra of two emitted electrons is confirmed, and the potential importance of multiple recollisions is discussed.
\end{abstract}

DOI: 10.1103/PhysRevLett.101.053001

Within the past three decades extensive studies on the interaction of intense laser fields with atoms and molecules have resulted in a profound understanding of various strong-field phenomena. Prominent examples are abovethreshold ionization (ATI) [1] or high-order harmonic generation [2], both essentially treatable within the single active electron (SAE) approximation. Correlated fewelectron processes, on the other hand, most important, for example, in nonsequential double (multiple) ionization (NSDI) (for a recent review see, e.g., [3]) have, until the present day, resisted any comprehensive modeling.

Recently, however, a breakthrough was achieved at high intensities $\left(\sim \mathrm{PW} / \mathrm{cm}^{2}\right)$ [4]. The application of many-particle imaging techniques [reaction microscopes and cold target recoil ion momentum spectroscopy (COLTRIMS)] [5] has allowed recording (multi)differential data on strong-field few-electron reactions and sophisticated calculations (see, e.g., [6] and references therein) have advanced their theoretical interpretation. As a result, a commonly accepted though simple picture has emerged, characterizing NSDI. Here an electron first tunnels into the field, is then accelerated, and finally thrown back onto its parent ion by the oscillating laser field. During "recollision" $n$-fold ionization might occur either in a direct $(e, n e)$-like encounter or indirectly via recollision-induced excitation of the ion plus subsequent field ionization (RESI) [7]. Signatures of the former are that both electrons are exclusively emitted into the same hemisphere along the polarization direction leading to "double-hump" shaped parallel momentum distributions of the ions (compensating the electron momenta). For RESI instead, according to the present understanding, the electrons can be emitted either parallel or back to back, thus filling the valley in between the double hump for the ions. Beyond the well-accepted simple scenario, however, major questions about the correlated electron emission are still far from being understood and are extensively investigated $[6,8]$ because recollision is at the very heart of attoscience, molecular tomography, or imaging [9].

At low intensities one intriguing though still widely unexplored question did arise early on within the above
PACS numbers: $32.80 . \mathrm{Rm}, 31.90 .+\mathrm{s}, 32.80 . \mathrm{Fb}, 32.80 . \mathrm{Wr}$

picture [10]. What happens when the energy of the recolliding electron of up to $3.17 U_{P}$ is not sufficient to directly ionize the parent ion or, at still lower energies, not even to reach its first excited state? $\left[U_{P}=\right.$ $\left(E_{0} / 2 \omega\right)^{2}$, ponderomotive potential; $E_{0}$, field amplitude; $\omega$, field frequency; atomic units (a.u.) are used throughout.] This is a situation where classical considerations do not hold any more and where one enters the fundamentally important nonperturbative multiphoton-multielectron regime. Still, multiple ionization rates were found to be dramatically enhanced due to electron-electron correlation [10].

One faces tremendous difficulties on the experimental side as well, since double ionization rates become prohibitively small at low intensities. Accordingly, only few differential studies have been reported on NSDI [11-15] close to threshold. In just one coincidence measurement [13], to the best of our knowledge, the energy of the recolliding electron was below the phase-dependent, field-modified ionization potential [12] $I_{P}^{*}=I_{P}-2\left(2\left|E_{0} \cos \left(\omega t_{r}\right)\right|\right)^{1 / 2}$, i.e., below the electron-impact ionization threshold $\left(I_{P}\right.$, ionization potential of the ion; $\omega t_{r}$, phase of the laser field where recollision occurs).

In this Letter, we report fully differential measurements for argon double ionization by 45 fs laser pulses at intensities as low as $4 \times 10^{13} \mathrm{~W} / \mathrm{cm}^{2}$. We thus penetrate deep into the multiphoton regime, with the maximum classical recollision energy of $3.17 U_{P}=7.4 \mathrm{eV}$ being well below both the unperturbed ionization (excitation) potential of $\mathrm{Ar}^{+}$of $27.6 \mathrm{eV}(13.5 \mathrm{eV})$ and the field-modified potential of $I_{P}^{*}=14.1 \mathrm{eV}$ [in the maximum of the field, i.e., for $\left.\cos \left(\omega t_{r}\right)=1\right]$. For single ionization we find well-known resonances very similar to earlier results at higher intensities. Surprisingly, however, the correlated electronmomentum $\left[P_{\|}\left(e_{1}\right)-P_{\|}\left(e_{2}\right)\right]$ spectra along the polarization ( II ) direction for double ionization reveal the dominance of back-to-back emission, never observed before. Moreover, a cutoff in the sum-energy spectrum of the emitted electrons at $\sim 5.3 U_{p}$ predicted below threshold [16] is confirmed, pointing to the importance of fieldassisted recollisions. Finally, the role of "accumulated 
rescattering excitation" as seen in quantum $[6,16]$ as well as in classical calculations [17] is discussed.

Since multiple-ionization probabilities rapidly drop with decreasing laser intensity, the small solid angles of traditional electron spectrometers as well as the low repetition rates (in the $\mathrm{kHz}$ regime) of conventional laser amplifiers both have established the main obstacles for coincidence experiments at intensities of interest. In order to overcome these limitations, a reaction microscope was combined with a long-cavity laser system based on a Herriott cell, allowing the generation of $800 \mathrm{~nm}, 45 \mathrm{fs}$, high-intensity pulses $\left(>10^{14} \mathrm{~W} / \mathrm{cm}^{2}\right)$ at a repetition rate of $6.2 \mathrm{MHz}$. The whole experimental setup is described in detail elsewhere [18]. In brief, the laser beam was focused onto the collimated supersonic gas jet in the ultrahigh vacuum chamber $\left(2 \times 10^{-10} \mathrm{mbar}\right)$. Created ions and electrons were guided towards two position-sensitive channel plate detectors by weak electric $(2 \mathrm{~V} / \mathrm{cm})$ and magnetic $(\sim 4.5 \mathrm{G})$ fields applied along the laser polarization axis achieving a $4 \pi$ detection solid angle for all particles of interest. From the time-of-flight and position on the detectors, the full momentum vectors of the recoil ions and electrons were calculated. Momentum resolutions for electrons as good as 0.02 a.u. along and 0.05 a.u. perpendicular to the spectrometer axis can be achieved (for details see [18]). Even though triple coincidences were recorded, we have not requested a three-particle coincidence for the generation of the correlation patterns in Fig. 2. Rather, the momentum of electron "two" was calculated from those of electron "one" and of the recoiling ion in order to avoid dead-time problems when two electrons hit the detector within less than $\sim 10$ ns. The spectra shown in Figs. 1(c) and 3 were deduced from the measured momenta of the two electrons, requesting the triple coincidence (sum-momentum resolution $\Delta P_{\text {sum }} \sim 0.1$ a.u.).

In order to highlight potentially important dynamic mechanisms and to compare our results to previously measured electron-ion coincidence spectra for Ar above the field-modified recollision threshold (at $100 \mathrm{TW} / \mathrm{cm}^{2}$ ) [13], we have performed appropriate cuts in our $4 \pi$ electron distributions for $70 \mathrm{TW} / \mathrm{cm}^{2}$ and integrated over the momenta of the ion as well as of the second electron [Figs. 1(a) and 1(b)]. For single ionization the coincident electron-momentum distributions [Fig. 1(a)] are quite similar at both intensities, clearly exhibiting a series of low-order ATI peaks, induced by so-called Freeman resonances [19]. They are discussed in some detail in [13] but are not the focus of this Letter. At higher energies, beyond the classical momentum limit of $2\left(U_{p}\right)^{1 / 2}$ in the so-called plateau regime, a series of highly intensity-sensitive structures arises in both experiments, which have been investigated in utmost detail in several previous experiments [20] for Ar and Xe. There is a large body of theoretical literature on the origin of these peaks (again beyond the scope of this Letter), discussing the relevance of doubly

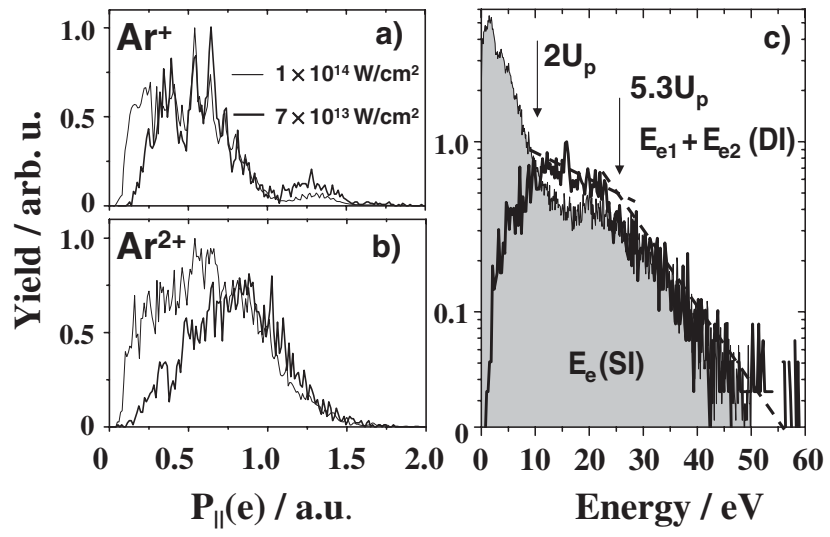

FIG. 1. (a),(b) Electron-momentum distributions along the polarization direction coincident with $\mathrm{Ar}^{+}$and $\mathrm{Ar}^{2+}$. Thick lines: Results at $7 \times 10^{13} \mathrm{~W} / \mathrm{cm}^{2}$ (45 fs). Thin lines: $1 \times$ $10^{14} \mathrm{~W} / \mathrm{cm}^{2}$ (100 fs) from [13]. (c) Sum energy of both emitted electrons for $\mathrm{Ar}$ double ionization at $7 \times$ $10^{13} \mathrm{~W} / \mathrm{cm}^{2}, 45 \mathrm{fs}$ (thick solid line). Shaded area: Electron energy distribution for Ar single ionization, scaled such that the high-energy tail overlaps with the double ionization spectrum for better comparison. Dashed lines: See text.

excited states [21] or of several mechanisms within the SAE. These include interference resonances at channel closings [20,22], multiphoton resonances involving excited states [20], the involvement of trapped dressed states [23], or of so-called "laser-induced states" close to the continuum [24]. The common bottom line, important for the discussion of the double ionization data presented here, may be summarized as follows: (i) multiple ionization or excitation plays little or no role for single ionization, and (ii) multiple elastic rescattering of electronic wave packets launched at different maxima of the pulse and interfering with each other are responsible for the observed effects.

With that in mind we now turn our discussion towards double ionization below the classical threshold intensity $I_{\text {th }}$ with the energy of the recolliding electron $E_{R}\left(\omega t_{r}\right)<I_{P}^{*}$. The ubiquitous, generally accepted signature for recollision-induced direct ionization (REDI) in an $(e, 2 e)$-like process is the occurrence of the "double hump" in the ion-momentum distribution [4]. Dynamically this means that the doubly charged ion is created close to a zero crossing of the electric field where the quivering electron comes back with about maximum energy of $3.17 U_{P}$ and, thus, receives a large drift momentum. Taking the perspective of the electrons, both collect large drift momenta as well (compensated by the ion), emerging into the same hemisphere along the laser polarization direction. Correlated electron-momentum plots along the parallel direction subsequently investigated in more refined experiments clearly observed this feature for $\mathrm{He}, \mathrm{Ne}, \mathrm{Ar}$ atoms $[4,7,8]$.

Thus, in order to trace the transition from above to below $I_{\text {th }}$ we first inspect correlated momentum spectra for Ar in 


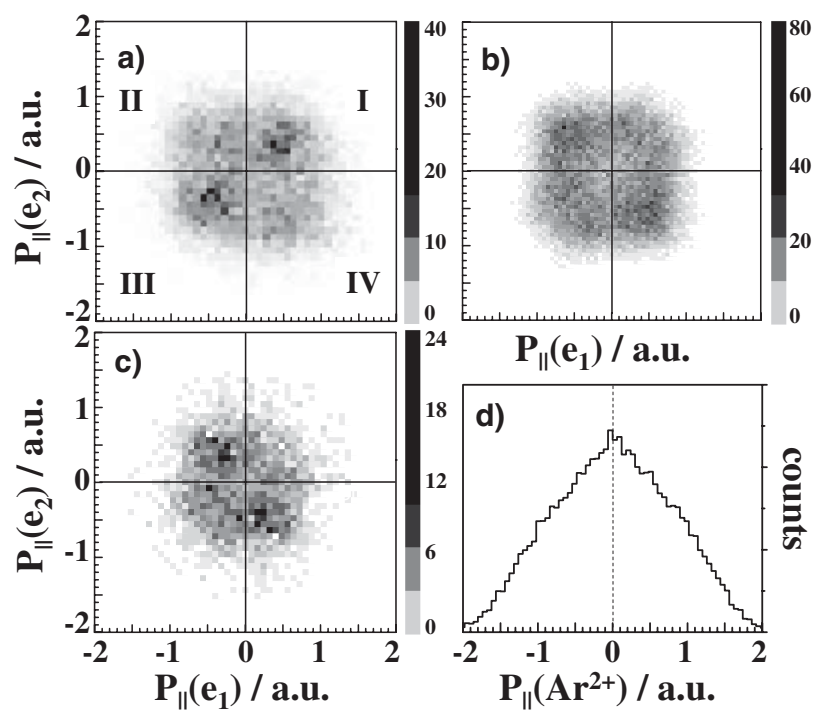

FIG. 2. Correlated longitudinal momentum spectra $P_{\|}\left(e_{1}\right)$ vs $P_{\|}\left(e_{2}\right)$ for $\mathrm{Ar}$ double ionization. (a) $9 \times 10^{13} \mathrm{~W} / \mathrm{cm}^{2}$ [11]. (b) $7 \times 10^{13} \mathrm{~W} / \mathrm{cm}^{2}$. (c) $4 \times 10^{13} \mathrm{~W} / \mathrm{cm}^{2}$. (d) Longitudinal momentum distribution of $\mathrm{Ar}^{2+}$ ions at $7 \times 10^{13} \mathrm{~W} / \mathrm{cm}^{2}$.

Fig. 2 for various intensities starting with previously measured data at $0.9 \times 10^{14} \mathrm{~W} / \mathrm{cm}^{2}$ [Fig. 2(a)], well above $I_{\text {th }}$. Here, most counts are found in quadrants 1 and 3 pointing to the dominance of REDI. In the RESI [7] scenario, the recolliding electron is assumed to excite the ion (with large $3 p$ to $3 d$ cross sections in Ar), and the excited electron then tunnels in one of the subsequent maxima of the laser field. The electrons are expected to be found (i) in any of the quadrants and (ii) with a maximum intensity around zero momentum for one of them, tunneling most likely close to a field maximum and, thus, acquiring little drift momentum. Consequently, within such a naïve RESI picture of independent tunneling after impact excitation, an unstructured correlation pattern with high intensity around $\left[P_{\|}\left(e_{1}\right), P_{\|}\left(e_{2}\right)\right]=(0,0)$ might be expected. Not really seen in Fig. 2(a) and also not in all previous experiments $[7,11]$, not much attention was paid to that fact until now, however, partly because the spectra were always blurred by REDI in quadrants 1 and 3.

Going close to and even below $I_{\text {th }}$ in Figs. 2(b) and 2(c), we surprisingly find, for the first time, (i) a dominance of events for electron emission into opposite hemispheres and (ii) a clear minimum at the origin, i.e., a strongly correlated emission of both electrons. The ion longitudinal momentum distribution [Fig. 2(d)] shows a sharp maximum at $P_{\|}\left(\mathrm{Ar}^{2+}\right)=0$, in contrast to the characteristic doublehump structure above the threshold [4,7].

The fact that the REDI contribution essentially disappears in quadrants 1 and 3 is in accordance with simple model-based expectations [7] as well as, e.g., with the predictions of classical calculations on the intensity dependence of a certain class of trajectories representing REDI [17]. The minimum at the origin might be due to mutual
Coulomb repulsion between the two electrons. This, however, should manifest itself in a similarly clear signature in the transverse correlated momentum spectra which is not observed as illustrated in Fig. 3. Here the transverse emission direction of one electron is fixed (along the arrow) and the $P_{x, y}$-momentum distribution of the second electron is inspected. In contrast to previous results [25], we do not find any indication of correlation in the transverse plane, conclusively discarding any effect of Coulomb repulsion.

In order to find a consistent explanation for the findings we have carefully analyzed three recent theoretical studies. First, the result of a $(1+1)$-dimensional quantum calculation for He at higher intensity [6] is most likely related with our work. The comparison seems to be justified since $\mathrm{He}$ and Ar have been shown to behave very similar in that for both of them excitation contributions are found to be larger than in Ne [7]. Surprisingly, a distinct pattern is observed for electrons emerging into opposite hemispheres (in addition to the well-known contribution of REDI in quadrants 1 and 3 at the higher intensity of $0.9 \mathrm{PW} / \mathrm{cm}^{2}$ ), which is very similar to what is observed in all experiments for Ar at any intensity. Here, the authors find that these quadrants are populated "later" during the laser pulse and assumed "the main contribution perhaps coming from accumulated rescattering excitations." Second, in classical calculations as well, another important class of trajectories was found, dominating double ionization at low intensities. Those have been shown to resemble continuous excitations during several recollisions leading to back-to-back emission of the electrons [17].

Third, and most importantly, calculations (and experiments) performed at the $390 \mathrm{~nm}$ well below (around) the threshold intensity demonstrated that field-assisted recollisions are responsible for He double ionization [16]. One signature for this mechanism being at work is the observation (at $390 \mathrm{~nm}$ as well as at $800 \mathrm{~nm}$ [26]) of an intensityindependent cutoff at $5.3 U_{p}$ in the sum-energy spectra of both emitted electrons for intensities below the threshold (the sum energy of $5.3 U_{p}$ is considered to be composed of

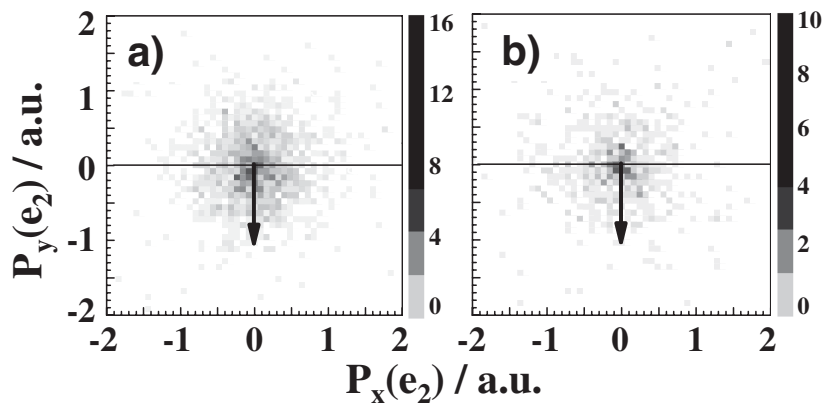

FIG. 3. Transverse momentum spectra $P_{x, y}\left(e_{2}\right)$ of electron $e_{2}$ for fixed emission direction of electron $e_{1}$ along the arrow $(7 \times$ $10^{13} \mathrm{~W} / \mathrm{cm}^{2}$ ). (a) Only events within quadrants 2 and 4 of Fig. 2(b) are selected. (b) Same as (a) but for quadrants 1 and 3 of Fig. 2(b). 
$\sim 1.9 U_{P}$ drift energy acquired by one "slow" electron and of $3.4 U_{P}$ delivered by another "fast," recolliding electron [26], as seen in Fig. 4 of [16]). As illustrated by the dashed lines in Fig. 1(c), guiding the eye, we find a clear signature of such a cutoff, thus, in essence, confirming the proposed mechanism.

In all three models, the dynamics of back-to-back emission $[6,17]$ or of the cutoff at $5.3 U_{p}$ [16] is essentially "captured in a simple model of $\mathrm{He}^{+}$ionization in which the rescattering collisions are treated as electric-field impulses that are too weak to significantly ionize the $\mathrm{He}^{+}(1 s)$ independently of the laser field" (as expressed in [16]).

One now remembers that the highly structured plateau part of the single ionization spectra was interpreted before to be due to repeated elastic recollisions and interference between the corresponding wave packets. These wave packets staying close to the nucleus in "trapped" or "laser-excited" states certainly can inelastically scatter as well with large probability, not included in any SAEbased calculation, thus, exciting the ion. Since they remain close to the ion, trapped in the combination of laser and Coulomb fields, they can most efficiently continue to absorb energy from the field and repeatedly excite the second electron with relatively large wave function overlap to higher and higher energies in a strongly correlated dynamic process. Actually, such a mechanism is at the heart not only of all three above-mentioned recent theoretical studies but, moreover, of the early "antenna model" [27].

Two further observations strongly support such an interpretation: First, using shorter pulses, i.e., reducing the number of recollisions the RESI mechanism was demonstrated to become less efficient [28], subsequent recollisional heating is suppressed. Second, the similarity in shape between the high-energy tails of the electron's sum-energy spectrum for $\mathrm{Ar}^{2+}$ and the $\mathrm{Ar}^{1+}$ ATI plateau [see Fig. 1(c)] is pointing to the intimate relationship between both reactions. Whereas in this picture the former would be caused by inelastic field-assisted recollisions, the latter was explained previously as being due to multiple elastic scattering of low-energy trapped and recolliding wave packets.

In summary, combining a novel high-repetition rate laser with a reaction microscope we succeeded in performing kinematically complete measurements on NSDI in the multiphoton regime, below the field-modified recollision threshold. We find a strongly correlated back-to-back emission of electrons, different from any observation before at higher intensities, a significant cutoff in the sum-electron energy spectrum at $5.3 U_{p}$, and discard Coulomb repulsion in the final state to be responsible for the observed longitudinal correlation. Comparison with the predictions of available quantum and classical calculations provides conclusive evidence that multiple inelastic field-assisted recollisions are responsible for our findings.

This research has been supported by the DFG within Contract No. Mo850/2. We thank K. Taylor and J. Parker for helpful discussions.

[1] P. Agostini et al., Phys. Rev. Lett. 42, 1127 (1979).

[2] A. L'Huillier and Ph. Balcou, Phys. Rev. Lett. 70, 774 (1993).

[3] A. Becker, R. Dörner, and R. Moshammer, J. Phys. B 38, S753 (2005).

[4] Th. Weber et al., Nature (London) 405, 658 (2000).

[5] J. Ullrich et al., Rep. Prog. Phys. 66, 1463 (2003).

[6] J. S. Prauzner-Bechcicki et al., Phys. Rev. Lett. 98, 203002 (2007).

[7] B. Feuerstein et al., Phys. Rev. Lett. 87, 043003 (2001).

[8] A. Staudte et al., Phys. Rev. Lett. 99, 263002 (2007); A. Rudenko et al., Phys. Rev. Lett. 99, 263003 (2007).

[9] P. B. Corkum and F. Krausz, Nature Phys. 3, 381 (2007).

[10] B. Walker et al., Phys. Rev. Lett. 73, 1227 (1994).

[11] E. Eremina et al., J. Phys. B 36, 3269 (2003).

[12] R. Wiehle and B. Witzel, Phys. Rev. Lett. 89, 223002 (2002).

[13] J.L. Chaloupka et al., Phys. Rev. Lett. 90, 033002 (2003).

[14] M. Weckenbrock et al., Phys. Rev. Lett. 92, 213002 (2004).

[15] J. Rudati et al., Phys. Rev. Lett. 92, 203001 (2004).

[16] J. S. Parker et al., Phys. Rev. Lett. 96, 133001 (2006).

[17] P. J. Ho et al., Phys. Rev. Lett. 94, 093002 (2005).

[18] Y. Liu et al., Opt. Express 15, 18103 (2007).

[19] R. R. Freeman et al., Phys. Rev. Lett. 59, 1092 (1987).

[20] M.P. Hertlein et al., J. Phys. B 30, L197 (1997); M. J. Nandor et al., Phys. Rev. A 60, R1771 (1999).

[21] P. H. Bucksbaum et al., J. Phys. B 30, L843 (1997).

[22] S. V. Popruzhenko et al., Phys. Rev. Lett. 89, 023001 (2002).

[23] H. G. Muller, Phys. Rev. Lett. 83, 3158 (1999).

[24] J. Wassaf et al., Phys. Rev. A 67, 053405 (2003).

[25] M. Weckenborck et al., Phys. Rev. Lett. 91, 123004 (2003).

[26] K. Taylor and J. Parker (private communication).

[27] M. Yu. Kuchiev, JETP Lett. 45, 404 (1987); J. Phys. B 28, 5093 (1995).

[28] A. Rudenko et al., Phys. Rev. Lett. 93, 253001 (2004). 